\title{
Association of major cardiovascular risk factors, risk factor clustering with cardiovascular diseases from eastern China A population-based cross- sectional study
}

Hua-Feng Yang

Nanjing CDC

Xin Hong ( $\square$ nj_hongxin@126.com )

Nanjing CDC

Wei-Wei Wang

Nanjing CDC

Qing Ye

Nanjing CDC

Zhi-Yong Wang

Nanjing CDC

Sheng-Xiang Qi

Nanjing CDC

Chen-Chen Wang

Nanjing CDC

Hai-Rong Zhou

Nanjing CDC

Chao Li

Nanjing CDC, Nanjing medical university

Zhen-Zhen Qin

Nanjing CDC

Research article

Keywords: Cardiovascular diseases, Risk Factors, Prevalence, Coronary heart disease, Stroke, China

Posted Date: December 23rd, 2019

DOl: https://doi.org/10.21203/rs.2.19393/v1

License: (c) (1) This work is licensed under a Creative Commons Attribution 4.0 International License.

Read Full License 
Page $2 / 21$ 


\section{Abstract}

Background: Cardiovascular diseases (CVDs) are serious public health issues and the burden of CVDs is at alarmingly high level in China. The aim of the current study was to estimate the prevalence of CVDs and modifiable risk factors, and explore the association of risk factors, risk factor clustering with CVDs among Nanjing adults from eastern China.

Methods: A population-based cross-sectional survey was conducted by a stratified clustered sampling between June and November 2011. A representative sample of 40,896 residents aged over 18 years was interviewed by face-to-face questionnaire survey, anthropometric measurements and laboratory examinations. The prevalence of CVDs including coronary heart disease (CHD) and stroke was determined according to a participant's self-report. Multivariable logistic regression models were used to estimate the relationship between relevant risk factors, their clustering and CVDs.

Results: The weighted prevalence of self-reported in the entire population was $1.1 \%$ for CHD; and $1.4 \%$ for stroke. The weighted prevalence of hypertension, diabetes, dyslipidemia, overweight or obesity, and current smoking was $25.0 \%, 6.7 \%, 29.6 \%, 35.9 \%$ and $24.5 \%$, respectively. In multivariable-adjusted models, higher levels of body mass index, systolic blood pressure and fasting plasma glucose could be associated with increased risks of CHD and stroke, whereas higher level of high-density lipoprotein cholesterol could be related to lower risks of CHD. Hypertension, diabetes and dyslipidemia were likely to be positively associated with self-reported CVDs regardless of genders in multivariable logistic regression models. Participants with CVDs had significant higher proportion of at least two of CVD risk factors than their counterparts without CVDs. Compared to those having no risk factor; adults with one, two, three or more CVD risk factors had possibly gradually increased risks of CHD and stroke in both genders.

Conclusion: Higher regional prevalence of CVDs was likely to be synergistic effects of risk factors aggregation. Therefore, multifactorial intervention strategies based on associated risk factors may be available for prevention and control of CVDs in Chinese population. Keywords: Cardiovascular diseases, Risk Factors, Prevalence, Coronary heart disease, Stroke, China

\section{Background}

Cardiovascular diseases (CVDs) are one of the most important public health issues in the world [1]. CVDs were the leading cause of deaths and loss of disability worldwide and were responsible for 17.5 million deaths in 2012, which accounted for $31.3 \%$ of global deaths [2]. Among these deaths, an estimated 7.4 million and 6.7 million were attributed to heart diseases and strokes, respectively. It is predicted that the overall number of CVD mortality will increase to 20 million by 2030 [3]. Additionally, more than $80 \%$ of cardiovascular deaths occurred in low- and middle-income countries [2]. The Global Burden of Disease study reported that coronary heart disease (CHD) and stroke were the most common types of CVDs [4].

With rapid economic growth, aging population, urbanization and westernization, the burden of CVDs is at alarmingly high level in China [5]. About 290 million individuals suffered from CVDs; in which 270 million 
had hypertension, 13 million were due to stroke, 11 million were attacked by CHD according to the 2016 report on cardiovascular diseases in China [6]. Since 2009, the CVDs mortality rate in rural areas has exceeded and consistently remained higher than that found in urban areas. CVDs were still the dominant cause of deaths among Chinese adults in 2015 , which contributed to $42.6 \%$ and $45.0 \%$ of all deaths in urban and rural areas, respectively [6].

The prevalence of major modifiable cardiovascular risk factors including hypertension, diabetes, overweight or obesity, dyslipidemia and tobacco use has been continuously increasing in China [7-12]. Moreover, it has been shown that CVD risk factors tend to cluster together in one individual [12-15]. CVD risk factor clustering will increase the incidence of CVDs and all-cause mortality significantly than a single risk factor $[10,11]$. The more the risk factors, the higher the risk of developing CVD events.

Stroke and CHD were ranked as the top two causes of deaths among Nanjing adults from the east of China since 2011 [16]. Nowadays there has been scant data regarding CVDs and modifiable risk factors in Chinese population. Consequently, the aim of the present study was to investigate the prevalence of CVDs and associated risk factors, and examine the relationship of CVDs with each risk factor and their clustering in large representative Nanjing adults.

\section{Methods}

\section{Study participants}

This population-based, cross-sectional study was carried out between June and November 2011 from the Chronic Disease and Risk Factor Surveillance in Nanjing, the capital of Jiangsu Province in east of China. The survey has been described in details previously [17]. Briefly, the inclusion criteria for sample population in our study were native permanent residents aged 18 years and above, who lived in local villages/communities for at least 6 months.

\section{Sample size and sampling}

According to the prevalence of diabetes among Jiangsu adults over 18 years of $5.8 \%$ [18], a of 0.05 , allowable error of $5 \%$, design effect of 1.5 and a non-response rate of $20 \%$, the required sample size was approximately 45,000 . A stratified clustered sampling method was used to select a representative sample in the general population. Firstly, 45 townships in 5 rural counties and 55 streets in 8 urban districts were all covered. Secondly, 3 administrative villages/neighborhood communities from each township/street were chosen based on probability proportion to size (PPS). Thirdly, a residential group with at least 150 households was randomly drawn by a simple random sampling from each chosen administrative village/neighborhood community. Finally, one eligible individual was randomly selected by a Kish grid from each chosen household. A total of 45,000 people were enrolled and invited to participant, and 41,072 agreed to take part in the survey (response rate: $91.3 \%$ ). One hundred and seventy-six subjects were excluded due to missing data on body weight $(n=28)$, plasma glucose $(n=26)$, smoking status 
$(n=38)$, self-reported CHD $(n=22)$, stroke $(n=26)$, or other covariates $(n=36), 40,896$ participants were included in the final analyses.

\section{Data Collection}

Data collection including questionnaire survey, anthropometric measurements and laboratory examinations was conducted face-to-face by trained interviewers according to a standard protocol at the local community healthcare centers/stations in the participants' residential area.

A structured questionnaire included demographic characteristics, behavior lifestyle, personal and family medical history of chronic diseases. Anthropometric measurements included height, body weight and blood pressure (BP). Body mass index (BMI) was calculated as weight (in kilograms) divided by the square of height (in meters). All participants were asked to rest for at least $5 \mathrm{~min}$ before BP measurements. BP was measured three times in a seated position by a trained staff using an automatic sphygmomanometer (OMRON HEM-7200, Japan). Three measurements were taken at 2-min intervals, and the mean of the last two readings was calculated. Blood samples were drawn between 7:00 am to 9:00 a.m. after a fasting overnight of at least 10 hours. Fasting plasma glucose (FPG) was assessed with a glucose oxidase method; triglycerides (TG), total cholesterol (TC), high-density lipoprotein cholesterol (HDL-C) and low-density lipoprotein cholesterol (LDL-C) were analyzed enzymatically with commercially available reagents.

A vigorous quality control was implemented to ensure the reliability of the investigation data. All staff members completed a uniform training program that taught the methods and procedures of the study. A manual of detailed instructions for administration of questionnaire survey, physical measurements and laboratory examinations was provided. Only certified staffs were allowed to collect data. All laboratory equipments were calibrated. All data were double entered and validated.

\section{Definition of variables}

\section{Self-reported CHD and stroke}

CHD was identified by the World Health Organization (WHO) clinical criteria (Interventional diagnosis: coronary arteriography) and the 10th revision of the International Classification of Diseases and Injuries (ICD-10) discharge diagnosis codes (ICD10: I20-I25). Stroke was also defined by the WHO as rapidly developing clinical signs of focal (or global) disturbance of cerebral function, with symptoms lasting 24 hours or longer, including hemorrhagic stroke (ICD-10: 160-161) and ischemic stroke (ICD-10: 163). The survey respondents with a history of $\mathrm{CHD}$ or stroke were asked to provide their medical records (inpatient or outpatient records) by the cardiologists or neurologists to confirm their declaration.

\section{CVD risk factors}

Five major CVD risk factors were defined as follows: (1) smoking status was determined by self-reported questionnaire. Each participant was classified as a current smoker, ex-smoker or nonsmoker. Current 
smokers: those who smoked at least one cigarette or equivalent on most days for at least 6 months at the time of the survey [19]. Ex-smoker smokers: those who had smoked for at least 6 months but were not smoking at the time of the survey. Both ex-smokers and nonsmokers were categorized as non-smokers in the present study. (2) Overweight or obesity: BMI $\geq 24.0 \mathrm{~kg} / \mathrm{m} 2$ [20]. (3) Hypertension: self-reported history of hypertension and/or currently undergoing antihypertensive treatment in the past two weeks, and/or the average systolic BP (SBP) $\geq 140 \mathrm{mmHg}$ and/or the average diastolic BP (DBP) $\geq 90 \mathrm{mmHg}$ in the field survey [21]. (4) Dyslipidemia: self-reported history of dyslipidemia and/or the use of antilipemic medication, and/or having at least one of the following in the field survey: TG $\geq 2.26 \mathrm{mmol} / \mathrm{L}, \mathrm{LDL}-\mathrm{C}$ $\geq 4.14 \mathrm{mmol} / \mathrm{L}, \mathrm{TC} \geq 6.22 \mathrm{mmol} / \mathrm{L}, \mathrm{HDL}-\mathrm{C}<1.04 \mathrm{mmol} / \mathrm{L}$ [22]. (5) Diabetes: self-reported history of diabetes and/or current treatment with antidiabetic medication (insulin or oral hypoglycemic agents), and/or FPG $\geq 7.0 \mathrm{mmol} / \mathrm{L}$ in the field survey [23]. One individual with two or more CVD risk factors was considered as having CVD risk factor clustering [10].

\section{Covariates}

Socioeconomic status (SES) was measured by education, occupation and total annual family income. The three variables were defined and categorized elsewhere [24]. Habitual dietary intakes over the previous year were estimated by the validated semi-quantitative food frequency questionnaire (FFQ) [25]. Subjects were defined as adopting unhealthy dietary habits if they had 3 or more of the following: insufficient intake of vegetables and fruits $(<400 \mathrm{~g} /$ day), insufficient intake of dairy products $(<300 \mathrm{~g} / \mathrm{day})$, insufficient intake of bean products (e.g., tofu, dry beans, bean sprouts, soy chicken, bean curd sheet, $<25 \mathrm{~g} /$ day), excessive intake of red meat (e.g., pork, beef and mutton, $>100 \mathrm{~g} /$ day) and excessive intake of salt ( $>6 \mathrm{~g} /$ day) [26-27]. Self-reported leisure-time physical activity $(P A)$ in the past week was assessed by the Chinese version of the International Physical Activity Questionnaire (IPAQ-C) [28]. The total PA time was calculated based on the total of the double time of vigorous activity per week and the time of moderate activity per week [29]. Participants were defined as having sufficient PA (the total time $\geq 150$ $\mathrm{min} /$ week) or having insufficient PA (the total time $<150 \mathrm{~min} /$ week) [29].

\section{Ethics approval}

Approval to conduct the study was granted by the academic and ethical committee of Nanjing Municipal Center for Disease Control and Prevention (Nanjing CDC), and written informed consent was obtained from all study participants prior to the survey.

\section{Statistical analysis}

Quantitative data were presented as means with standard deviation (SD), and qualitative data as proportions with $95 \%$ confidence interval (Cl). Differences in quantitative variables and qualitative variables were compared by independent $t$ test and Chi-square test, respectively. Standardized values (means, prevalence) were calculated using the weight coefficients to represent the total Nanjing adult population aged $\geq 18$ years. Weight coefficients accommodated the sampling scheme for unequal probabilities of sample selection, as well as the post-stratification weights, which harmonized the 
standard population of the 2009 Nanjing Sixth National Population Census by two genders (men and women) and 12 age groups (18-19 years, 20-24 years, 25-29 years, 30-34 years, 35-39 years, 40-44 years, $45-49$ years, 50-54 years, 55-59 years, 60-64 years, 65-69 years and $\geq 70$ years). Multivariable logistic regression models were used to examine the association of each risk factor, CVD risk factor clustering with self-reported CHD and stroke for men and women separately. Models were adjusted for age only (model 1), age plus all other major CVD risk factors (model 2), and all variables in model 2 plus marriage, residence, SES, family history of CVDs, physical activity and dietary habits (model 3). Statistical analyses were conducted by the SPSS software (version 20; IBM, Armonk, NY, USA). All P-values were two-tailed with a significant level of $<0.05$.

\section{Results}

\section{Socio-demographic characteristics}

Of 40,896 participants, more than half of the participants were women ( $54.7 \%)$. The mean age was 48.4 \pm 16.4 years (range $18-86$ years). The majority of residents lived in rural areas $(59.5 \%)$. Almost of adults were married or living with a partner (84.5\%), nearly one third of participants had education of primary school or below (30.3\%) and had high level of annual family income (31.3\%). The percentage of participants with unhealthy dietary habits and insufficient PA were $73.3 \%$ and $63.3 \%$, respectively.

Prevalence of cardiovascular diseases and five major CVD risk factors

The weighted prevalence of self-reported CHD in the general population was $1.1 \%(1.2 \%$ in men and $0.9 \%$ in women), which was lower than that of stroke as $1.4 \%$ (1.7\% in men and $1.2 \%$ in women) (Table 1 ). There were significant increased trends of prevalence with age for both CHD and stroke regardless of genders. In addition, the weighted prevalence of cardiovascular diseases was higher in men than that in women for each age groups (all $\mathrm{P}<0.01$ ).

The standardized prevalence of hypertension, diabetes, dyslipidemia, overweight or obesity, and current smoking of total participants was $25.0 \%, 6.7 \%, 29.6 \%, 35.9 \%$ and $24.5 \%$, respectively (Table 1 ). The weighted prevalence of these risk factors was more common in men than in women (all $P<0.001$ ), especially for current smoking. CVD risk factor prevalence increased significantly with age either in men or women (all $\mathrm{P}<0.001$ ).

Association of CVD risk factor level with cardiovascular diseases

Table 2 showed that the average standardized levels of BMI, SBP, DBP, FPG, TC, TG, LDL-C were significantly higher among subjects with $\mathrm{CHD}$ or stroke, while the mean weighted value of HDL-C level was higher in those without CVDs conversely (all P< 0.001 ).

After adjustment for age (model 1), higher levels of BMI, SBP, DBP, FPG, TC and TG had possibly increased risks of CHD; in contrast, participants with higher value of HDL-C were likely to be at lower risks 
of CHD. Similarly, higher levels of BMI, SBP, DBP, FPG, TC, TG and LDL-C may increase risks of stroke (model 1). Higher values of BMI, SBP and FPG could be associated with increased risks of CHD and stroke, whereas higher level of HDL-C could be related to lower risks of CHD in model $3($ all P $<0.05)$ (Table 3).

Association of CVD risk factors with cardiovascular diseases

In age-adjusted analyses (model 1), hypertension, diabetes and dyslipidemia were possibly to be positively associated with self-reported CHD for both genders (Table 4). Current smoking could be associated with CHD in men, whereas overweight or obesity could be associated with CHD among women. Associations of CVD risk factors with CHD were attenuated and lost statistical significance in model 2 or in model 3.

In model 1, hypertension, diabetes, dyslipidemia, overweight or obesity was likely to be associated with self-reported stroke for men and women. With further adjustment for other CVD risk factors (model 2) and for variables in model 3 , the association of hypertension, diabetes and dyslipidemia with stroke was attenuated but remained significant regardless of genders, respectively.

Association of CVD risk factor clustering with cardiovascular diseases

Overall, $34.1 \%, 29.8 \%$ and $36.1 \%$ of total samples had none, one and at least two of CVD risk factors, respectively. Men and the elderly were more likely to be CVD risk factor clustering than women and the younger counterparts (all $P<0.01$ ). The proportion of none, one, two or more CVD risk factors was $4.3 \%$, $17.9 \%$ and $77.8 \%$ for self-reported CHD participants (Fig. 1-A), 4.3\%, 16.3\% and 79.4\% for self-reported stroke adults, respectively (Fig. 1-B). Compared to those without CVDs, Participants with CHD or stroke had significant higher proportion of two or more CVD risk factors and lower percentage of no risk factor for the entire study population and both genders (all $\mathrm{P}<0.01$ ).

After adjustment for age (model 1), compared with those having no risk factor, men with one, two, three or more CVD risk factors had possibly gradually increased risks of CVDs; the age-adjusted odds ratios (ORs) for CHD were 2.304, 3.394 and 6.197; the corresponding values for stroke were 3.467, 4.867 and 11.352, respectively. Similarly, compared to their counterparts without risk factor, the adjusted ORs for CHD in women having one, two, at least three of CVD risk factors were 3.673, 4.480 and 9.730. The adjusted ORs for stroke associated with the same risk levels were 1.595, 4.080 and 6.462, respectively. With further adjustment for other factors (model 2), the association between CVD risk factor clustering and CVDs was attenuated but remained significant regardless of genders (Table 5).

\section{Discussion}

The cross-sectional study obtained regional prevalence of CVDs and associated risk factors among representative population of Nanjing adults. Participants with CVDs had higher levels of BMI, BP, FPG, TG, TC, LDL-C and a lower HDL-C level than subjects without CVDs. We also found that five modifiable risk 
factors could be associated with CVD events, and risk factors clustering had possibly increase gradually risks of self-reported CHD or stroke regardless of genders.

The overall weighted prevalence of self-reported CHD and stroke in the present study was higher than that in the nationwide population [13], but lower than in the United States [31]. A nationally representative sample of 46,239 adults aged $\geq 20$ years from the 2007-2008 China National Diabetes and Metabolic Disorders Study indicated that the standardized prevalence of self-reported CHD and stroke was $0.6 \%$, 0.8\%, respectively [13]. In 2008-2011 multicenter prospective population-based Hispanic Community Health Study [31], the overall prevalence of CHD and stroke among persons aged 18 to 74 years was $4.2 \%$ and $2.0 \%$ for men, and $2.4 \%$ and $1.2 \%$ for women, respectively.

Sino-Monitoring Trends and Determinants in Cardiovascular Disease (Sino-MONICA) project [32, 33], the China Multicenter Collaborative Study conducted from 1987 to 1993, indicated that stroke incidence and mortality were higher compared with western countries, but the same rates for CHD were lower than western countries. The pattern of CVDs occurrence in China was the high stroke events combined with low CHD events, which was similar to other eastern Asian countries (Korea, Japan, Singapore and Thailand). The epidemic pattern was thought to be associated with higher BP levels and lower serum TC levels as seen in Sino-MONICA study [33].

In previous studies [6,32,33], the morbidity and mortality of CVDs in China was significantly higher in northern and rural areas than southern and urban counterparts, respectively. The geographic variations in CVD mortality and incidence may attribute to the north-south differences in CVD risk factor profiles. Dietary preferences of salt or other spices in northern China were related to the higher prevalence of hypertension. It has been estimated that 1.7 million annual cardiovascular deaths have been due to excess salt/sodium intake (more than $5 \mathrm{~g}$ per day) globally in 2010 [34]. High salt consumption is related to raised blood pressure and increased risk of CVD events. Furthermore, the cold weather may limit outdoor physical activity, enhance fat reservation, and consequently increase the risk of obesity and metabolic abnormalities in northern regions. Additionally, increasing urbanization, limited medical resources and imperfect healthcare system may be responsible for the higher prevalence of CVDs and its risk factors in rural regions.

Our findings revealed that higher levels of BMI, SBP and FPG could be associated with increased risks of self-reported CHD and stroke, whereas higher level of HDL-C could be related to lower risks of CHD. In addition, hypertension, diabetes and dyslipidemia had possibly positively associations with the risk of CVDs, which was consistent with previous observations [7-12]. In China, the five leading risk factors were high blood pressure, tobacco smoking, high sodium intake, high FPG and high BMI as reported the 2013 Global Burden of Disease Study [4]. Although the prevalence of CVD risk factors has decreased in economically developed countries, the corresponding prevalence has increased in economically developing countries, including China. Hypertension, as one of the most important risk factor for CVDs, has been found to be highly prevalent in China currently. According to the 2002 the China National Nutrition and Health Survey (NNHS) [35], the prevalence of hypertension among Chinese adults aged over 
18 years was $18.8 \%$, and increased to $25.2 \%$ in the 2015 report of China National Nutrition and Chronic Disease Survey (NNCDS) [36], which was close to our finding (25.0\%). Based on data from 2013 report on chronic disease risk factor surveillance in China [37], the awareness (40.9\%), treatment (32.5\%) and control $(9.7 \%)$ of hypertension were still suboptimal. A reduction of $10 \mathrm{mmHg}$ in SBP was connected with a $22 \%$ reduction in $\mathrm{CHD}$ and $41 \%$ reduction in stroke in randomized trials [38]. The overall prevalence of diabetes was estimated to be $11.6 \%$ in the Chinese adult aged 18 years and older from the 2010 China Chronic Disease and Risk Factor Surveillance [39], whereas the rate was 2.6\% in 2002 [35]. The prevalence of dyslipidemia (34.0\%) among Chinese adults was high, but rates of awareness (31.0\%), treatment $(19.5 \%)$ and control (8.9\%) of dyslipidemia were low in the general Chinese population aged more than 18 years between 2007 and 2010 [40]. The 2015 NNCDS reported [36] that the prevalence of overweight and obesity reached $30.1 \%$ and $11.9 \%$, whereas the corresponding value was $17.6 \%$ and $5.6 \%$ in the 2002 NNHS [35], respectively. In the 2010 Global Adult Tobacco Survey [41], the overall smoking rate among Chinese adults over 15 years of age was $28.1 \%$ ( $52.9 \%$ of men and $2.4 \%$ of women). The rate of diabetes, dyslipidemia, overweight or obesity, current smoking in the present study was lower than the national levels mentioned above [36, 39-41].

Our finding also indicated that the risks for self-reported CHD and stroke were likely to increase substantially with each additional risk factor, in line with other previous reports [13, 42, 43]. Numerous studies $[12,42,43]$ have confirmed that CVD incidence and all-cause mortality increased markedly with risk factor clustering. Data from the First National Health and Nutrition Examination Surveys Epidemiologic Follow-up Study [42], the adjusted relative risks of $\mathrm{CHD}$ in adults with one, two, three, four or more risk factors were 1.6, 2.2, 3.1 and 5.0 during 21 years of follow-up, respectively, compared to those with no risk factors. The adjusted relative risks of stroke associated with the same risk levels were $1.4,1.9,2.3$ and 4.3, respectively. After 26 years of follow-up, favorable cardiovascular risk profile in middle age was associated with better quality of life and lower risk of CVDs in older age from the Chicago Heart Association Detection Project in Industry [43]. By applying the recalibrated Framingham risk function to the Inter Asia study [12], the 10-year CHD risk increased 0.2\%, 1.7\%, 3.2\% and 10.4\%, respectively, for Chinese adults with none, one, two and at least three of risk factors. A prospective cohort study [44] carried out in Chinese population aged 35-64 years showed that the incidence rate of CVDs increased significantly with the number of risk factor clustering, and found that there were a significant synergistic effects between each CVD risk factor.

Proper management including lifestyle modification or medical treatment may be the most effective strategy in reduction the pervasive CVDs burden under the supervision of the government. At the national health conference in 2016, China government ensured that health became an explicit national political priority with the approval of the Healthy China 2030 Planning Outline by China's Central Party Committee and the State Council. This document was the first general guideline for the promotion of healthy lifestyles and physical fitness at the national level, which stressed that the health should be combined into all major policies. 
First, China has launched national fitness program vigorously and promoted comprehensive development of both recreational and competitive sports in recent years. Sufficient physical activity can reduce the risk of cancer, diabetes, CVDs and all-cause mortality [45]. To meet the requirement of growing health, government has designated 8th August as "national fitness day" since 2009. In 2013, only $15 \%$ of Chinese residents aged over 18 years took regular exercise (participating moderate or vigorous intensive PA at least 10 minutes three days per week) in leisure time [37]. The percentage of regular physical activity among Chinese adults will increase to $40 \%$ in 2030 . Second, Chinese adults are recommended to adopt healthy dietary pattern, with high intake of whole grains, fresh vegetables and fruits, dairy products, soy foods, poultry meat, fish and shrimp, unsaturated cooking oil, and low intake of processed meat, red meat, fried foods, western fast foods, snacks and desserts [46]. Meanwhile, reduction in salt intake is also an essential part of CVDs management strategy. The average daily intake of salt in 2012 was $10.5 \mathrm{~g}$ per person [36], which was much higher than the recommended level by 2016 dietary guidelines for Chinese residents (lee than $6 \mathrm{~g} / \mathrm{d}$ ). The daily intake of salt in 2030 will be reduced relatively by $20 \%$ compared to 2012 national level. Third, China has tightened rules on smoking in public places. The rule that smoking is banned in indoor public places has been in force since 1 May 2011. The Ministry of Education and Ministry of Health jointly issued guidelines with regard to the further enhancement of tobacco control in schools in June 2011. All indoor and outdoor areas of kindergartens, primary and secondary schools, including vocational schools, should be smoke-free. Additionally, the overall prevalence of smoking among Chinese adults aged over 15 years will reduce to $20.0 \%$ in 2025. Fourth, with the increasing prevalence of hypertension, diabetes, dyslipidemia and obesity in China, better management of CVD risk factors are in urgently needed. Due to the poor public awareness, treatment and control of risk factors, more education and training (e.g., increased government investment), the application of evidence-based screening, prevention and therapies should be encouraged in China [6].

Several limitations of our study should be noted. Firstly, this study was a single-center, observational study. The associations between CVD risk factors and CVD events should be evaluated in the future prospective studies from multiply centers. Secondly, the information on smoking status, CHD and stroke status was based on self-reported data, which could be subject to reporting bias and may underestimate the prevalence of CVDs. To ensure the reliability of these self-reported measures, the survey respondents with a history of $\mathrm{CHD}$ or stroke were asked to provide investigators with their medical records in tertiary hospitals. Thirdly, CVD risk factors were involved modifiable and non-modifiable risk factors, only five major modifiable factors were considered in the present study. However, other relative risk factors (older age, men, lower levels of SES, having family history of CVDs, physical inactivity and unhealthy dietary habits) were taken into account to enter into the logistic regression analyses. Finally, cross-sectional studies with binary outcomes analyzed by logistic regression are frequent in the epidemiological literature. There is nothing intrinsically wrong with them. However, the ORs can importantly overestimate the prevalence ratio. Therefore, the different alternatives to analyze our data using prevalence ratios instead of ORs can be chosen for the future.

The present study also had several strengths. This was the first study to explore the association of CVD risk factors, their clustering with CVDs among a large representative sample of Nanjing adults. Another 
strength was that a strict quality control was implemented to ensure the reliability and validity of study data, which suggested that the findings could be cautiously generalized to other population.

\section{Conclusions}

In conclusion, CVD risk factors and their clustering were widespread among adults over 18 years in Nanjing from eastern China. Higher regional prevalence of CVDs could be attributable to combined effects of associated risk factors clustering. Population-wide and cost-effective interventions, such as enhancement government investment in education programs, adopting healthy lifestyle behaviors, better management of CVD risk factors, improvement the coverage of drug therapy of CVDs, as integrated primary care programs to high-risk individuals, may be implemented to prevent and control of CVDs in the future.

\section{Abbreviations}

CVD:cardiovascular disease, CHD:coronary heart disease, PPS:probability proportion to size, BP:blood pressure, BMI:body mass index, FPG:fasting plasma glucose, TG:triglycerides, TC:total cholesterol, LDLC:Iow-density lipoprotein cholesterol, HDL-C:high-density lipoprotein cholesterol, ICD-10:International classification of diseases, 10th revision, SBP:systolic blood pressure, DBP:diastolic blood pressure, SES:socio-economic status, FFQ:food frequency questionnaire, PA:physical activity, IPAQ:International Physical Activity Questionnaire, CDC:Center for Disease Control and Prevention, OR:odds ratio, 95\% Cls: 95\% confidence intervals, SD:standard deviation.

\section{Declarations}

\section{Acknowledgements}

We are grateful to all the dedicated fieldworkers who took part in the surveys and all participants who facilitated the survey implementation at each community.

\section{Funding}

This research work was funded by the Nanjing Municipal Medical Science and Technique Development Foundation, China (grant no. 2007-ZKX07026). The funder had no role in the decision to collect data, data analysis, or reporting of the results.

\section{Authors' contributions}

The authors' contributions are as follows: $\mathrm{XH}, \mathrm{HFY}$ and QY conducted data management, statistical analysis and design, and writing; HFY, WWW, ZYW, SXQ, CCW, HRZ, CL and ZZQ conducted investigation 
and data collection; $\mathrm{XH}, \mathrm{HFY}$ and WWW contributed to editing and proof reading of the final version of the manuscript. All authors read and approved the final version of the manuscript.

\section{Ethics approval}

Approval to conduct the study was granted by the academic and ethical committee of Nanjing Municipal Center for Disease Control and Prevention (Nanjing CDC), and written informed consent was obtained from all study participants prior to the survey.

\section{Competing interests}

The authors declare that they have no competing interests.

\section{References}

1.

Lloyd-Jones D, Adams R, Carnethon M, et al American Heart Association Statistics Committee and Stroke Statistics Subcommittee. Heart disease and stroke statistics-2009 update: a report from the American Heart Association Statistics Committee and Stroke Statistics Subcommittee. Circulation. 2009;119:4806.

2.

World health organization

Global status report on noncommunicable diseases 2014 [EB/OL]

World health organization. Global status report on noncommunicable diseases 2014 [EB/OL]. [2016-0809]. http://www.who.int/nmh/publications/ncd- status-report-2014/en/.

3.

Mathers CD, Loncar D. Projections of global mortality and burden of disease from 2002 to 2030 . PLoS Med. 2006;3(11):e442.

4.

GBD 2013 Mortality and Causes of Death Collaborators. Global, regional, and national age-sex specific all-cause and cause-specific mortality for 240 causes of death, 1990-2013: a systematic analysis for the Global Burden of Disease Study 2013. Lancet. 2015;385(9963):117-71.

5 .

Stevens W, Peneva D, Li JZ, et al. Estimating the future burden of cardiovascular disease and the value of lipid and blood pressure control therapies in China. BMC Health Serv Res. 2016;16:175.

6.

National center for cardiovascular disease, China. Report on cardiovascular disease in China, 2016. Encyclopedia of China publish house,2017. (In Chinese).

7. 
Xu S, Jiayong Z, Li B, et al. Prevalence and Clustering of Cardiovascular Disease Risk Factors among Tibetan Adults in China: A Population-Based Study. PLoS One. 2015;10:e0129966.

8.

$\mathrm{Ni} \mathrm{W}$, Weng R, Yuan X, et al. Clustering of cardiovascular disease biological risk factors among older adults in Shenzhen City, China: a cross-sectional study. BMJ Open. 2019;9(3):e024336.

9.

Li G, Guo G, Wang W, et al. Association of prehypertension and cardiovascular risk factor clustering in Inner Mongolia: a cross-sectional study. BMJ Open. 2017;7(6):e015340.

10.

Yu J, Ma Y, Yang S, et al. Risk Factors for cardiovascular disease and their clustering among Adults in Jilin (China). Int J Environ Res Public Health. 2015;13:ijerph13010070.

11.

Wu J, Cheng X, Qiu L, et al. Prevalence and clustering of major Cardiovascular Risk Factors in China: A Recent Cross-Sectional Survey. Med (Baltim). 2016;95:e2712.

12.

Gu D, Gupta A, Muntner P, et al. Prevalence of cardiovascular disease risk factor clustering among the adult population of China: results from the International Collaborative Study of Cardiovascular Disease in Asia (InterAsia). Circulation. 2005;112:658-65.

13.

Yang ZJ, Liu J, Ge JP, et al, China National Diabetes and Metabolic Disorders Study Group. Prevalence of cardiovascular disease risk factor in the Chinese population: the 2007-2008 China National Diabetes and Metabolic Disorders Study. Eur Heart J. 2012;33:213-20.

14.

Khanal MK, Mansur Ahmed MSA, Moniruzzaman M, et al. Prevalence and clustering of cardiovascular disease risk factors in rural Nepalese population aged 40-80 years. BMC Public Health. 2018;18(1):677. 15.

D'Agostino RB, Sr, Vasan RS, Pencina MJ, et al. General cardiovascular risk profile for use in primary care: the Framingham Heart Study. Circulation. 2008;117(6):743-53.

16.

Yang HF, Chen XP, Hong X, et al. Influence of epidemiological trend of disease mortality on life expectancy among residents in Nanjing from 2011 to 2014. Jiangsu Journal of Preventive Medicine. 2017;28:512-4. (In Chinese).

17.

Hong X, Ye Q, He J, et al. Prevalence and clustering of cardiovascular risk factors: a cross-sectionalsurvey among Nanjing adults in China. BMJ Open. 2018;8(6):e020530.

18.

Dai Y, Yuan BJ, Shi ZM. A cross-sectional study on diabetes mellitus among adults of 18 years old and above in Jiangsu Province. Journal of Hygiene Research. 2010;39:626-7. (In Chinese).

19. 
World Health Organization. Guidelines for the conduct of tobacco smoking surveys for the general population. Technical Document No. WHO/SMO/83.4.Geneva:WHO,1983.

20.

Zhou B. Predictive values of body mass index and waist circumference to risk factors of related diseases in Chinese adult population. Zhonghua Liu Xing Bing Xue Za Zhi. 2002;23:5-10. (In Chinese).

21.

James PA, Oparil S, Carter BL, et al. 2014 evidence-based guideline for the management of high blood pressure in adults: report from the panel members appointed to the Eighth Joint National Committee (JNC 8). JAMA. 2014;311(5):507-20.

22.

Joint Committee for Developing Chinese guidelines on Prevention and Treatment of Dyslipidemia in Adults. Chinese guidelines on prevention and treatment of dyslipidemia in adults. Zhonghua Xin Xue Guan Bing Za Zhi. 2007;35:390-419. (In Chinese).

23.

American Diabetes Association. Diagnosis and classification of diabetes mellitus. Diabetes Care. 2013;36(Suppl 1):67-74.

24.

Xu F, Yin XM, Wang Y. The association between amount of cigarettes smoked and overweight, central obesity among Chinese adults in Nanjing, China. Asia Pac J Clin Nutr. 2007;16:240-7. 25.

Hong $X, Y e Q$, Wang Z, et al. Reproducibility and validity of dietary patterns identified using factor analysis among Chinese populations. Br J Nutr. 2016;116(5):842-52.

26.

Neumann Al, Martins IS, Marcopito LF, et al. Dietary patterns associated with risk factors for cardiovascular disease in a Brazilian city. Rev Panam Salud Publica. 2007;22(5):329-39.

27.

Whitton C, Rebello SA, Lee J, et al. A Healthy Asian A Posteriori Dietary Pattern Correlates with A Priori Dietary Patterns and Is Associated with Cardiovascular Disease Risk Factors in a Multiethnic Asian Population. J Nutr. 2018;148(4):616-23.

28.

Deng HB, Macfarlane DJ, Thomas GN, et al. Reliability and Validity of the IPAQ-Chinese: The Guangzhou Biobank Cohort Study. Med Sci Sports Exerc. 2008;40:303-7.

29.

U.S. Department of Health and Human Services. 2008 Physical Activity Guidelines for Americans. Available: http://www.health.gov/paguidelines. Accessed on May 20, 2011.

30.

Zhang L, Wang F, Wang L, et al. Prevalence of chronic kidney disease in China: a cross-sectional survey. Lancet. 2012;379:815-22.

31. 
Daviglus ML, Talavera GA, Avilés-Santa ML, et al. Prevalence of major cardiovascular risk factors and cardiovascular diseases among Hispanic/Latino individuals of diverse backgrounds in the United States. JAMA. 2012;308:1775-84.

32.

Wu Z, Yao C, Zhao D, et al. Sino-MONICA project: acollaborative study on trends and determinants in cardiovascular diseases in China, Part i: morbidity and mortality monitoring. Circulation. 2001;103(3):462-8.

33.

Wu Z, Yao C, Zhao D, et al. Cardiovascular disease risk factor levels and their relations to CVD rates in China-results of Sino-MONICA project. Eur J Cardiovasc Prev Rehabil. 2004;11(4):275-83. 34.

Mozaffarian D, Fahimi S, Singh GM, et al. Global Burden of Diseases Nutrition and Chronic Diseases Expert Group. Global sodium consumption and death from cardiovascular causes. N Engl J Med. 2014;371(7):624-34.

35.

Li L. China National Nutrition and Health Survey 2002: Hypertension. Beijing: People's Medical Publishing House; 2005. (In Chinese).

36.

National Commission of Health Bureau of disease control and Prevention. Report on China national nutrition and chronic disease survey. Beijing: People's Medical Publishing House; 2015. 2015. (In Chinese).

37.

National Center for Chronic and Non-communicable Disease Control and Prevention, Chinese Center for Disease Control and Prevention. Report on Chronic Disease Risk Factor Surveillance in China, 2013. Beijing: Military Medical Science Press; 2016. (In Chinese). 38.

Law MR, Morris JK, Wald NJ. Use of blood pressure lowering drugs in the prevention of cardiovascular disease: meta-analysis of 147 randomized trials in the context of expectations from prospective epidemiological studies. BMJ. 2009;338:b1665.

39.

Xu Y, Wang L, He J, et al. 2010 China Noncommunicable Disease Surveillance Group. Prevalence and control of diabetes in Chinese adults. JAMA. 2013;310:948-59.

40.

Pan L, Yang Z, Wu Y, et al. The prevalence, awareness, treatment and control of dyslipidemia among adults in China. Atherosclerosis. 2016;248:2-9.

41.

Li Q, Hsia J, Yang G. Prevalence of smoking in China in 2010. N Engl J Med. 2011;364:2469-70. 42.

Yusuf HR, Giles WH, Croft JB, et al. Impact of multiple risk factor profiles on determining cardiovascular disease risk. Prev Med. 1998;27:1-9. 
43.

Daviglus ML, Liu K, Pirzada A, et al. Favorable cardiovascular risk profile in middle age and health-related quality of life in older age. Arch Intern Med. 2003;163:2460-8.

44.

Wu Z, Yao C, Zhao D, et al. A prospective cohort study on cardiovascular disease incidence in 11 provinces in China âष冈. Associations between individual risk factor aggregation and cardiovascular disease incidence. Chin J Cardiol. 2001;29:246-49. (In Chinese).ãष区.

45.

Global recommendations on physical activity for health. Geneva: World Health Organization; 2010 (http://whqlibdoc.who.int/publications/2010/9789241599979_eng.pdf, accessed 4 November 2014). 46.

Wang D, He Y, Li Y, et al. Joint Association of Dietary Pattern and Physical Activity Level with Cardiovascular Disease Risk Factors among Chinese Men: A Cross-Sectional Study. PLoS One. 2013;8(6):e66210.

\section{Tables}

Table 1

The weighted prevalence $(95 \% C I)$ of CVDs and five major risk factors among Nanjing sample $(n=40,896)$.

\begin{tabular}{|c|c|c|c|c|c|c|c|}
\hline Groups & CHD & Stroke & Hypertension & Diabetes & Dyslipidemia & Overweight or obesity & Current smoking \\
\hline Total & $1.1(1.0,1.2)$ & $1.4(1.3,1.5)$ & $25.0(24.6,25.4)$ & $6.7(6.5,6.9)$ & $29.6(29.2,30.0)$ & $35.9(35.4,36.4)$ & $24.5(24.1,24.9)$ \\
\hline \multicolumn{8}{|l|}{ Men } \\
\hline 18-34 years & $0.5(0.3,0.8)$ & $0.7(0.5,1.0)$ & $7.6(6.8,8.4)$ & $2.8(2.3,3.3)$ & $23.8(22.6,25.0)$ & $28.8(27.5,30.1)$ & $32.0(30.7,33.3)$ \\
\hline $35-59$ years & $0.8(0.6,1.0)$ & $0.9(0.7,1.1)$ & $30.8(29.8,31.8)$ & $7.4(6.9,7.9)$ & $38.0(37.0,39.0)$ & $48.0(47.0,49.0)$ & $59.3(58.3,60.3)$ \\
\hline$\geq 60$ years & $3.3(2.8,3.9)$ & $5.7(5.1,6.4)$ & $58.4(57.0,59.8)$ & $15.5(14.5,16.5)$ & $37.0(35.7,38.3)$ & $43.6(42.2,45.0)$ & $45.0(43.6,46.4)$ \\
\hline Overall & $1.2(1.1,1.4)$ & $1.7(1.5,1.9)$ & $26.6(26.0,27.2)$ & $7.0(6.6,7.4)$ & $32.2(31.5,32.9)$ & $39.7(39.0,40.4)$ & $46.1(45.4,46.8)$ \\
\hline \multicolumn{8}{|l|}{ Women } \\
\hline 18-34 years & $0.1(0.04,0.2)$ & 0.0 & $3.8(3.3,4.3)$ & $1.5(1.2,1.8)$ & $15.1(14.2,16.0)$ & $14.0(13.1,14.9)$ & $0.5(0.3,0.7)$ \\
\hline $35-59$ years & $0.6(0.5,0.8)$ & $0.6(0.5,0.8)$ & $25.2(24.4,26.0)$ & $6.8(6.3,7.3)$ & $31.1(30.2,32.0)$ & $40.7(39.8,41.6)$ & $1.5(1.3,1.7)$ \\
\hline$\geq 60$ years & $3.1(2.7,3.6)$ & $4.8(4.3,5.4)$ & $58.6(57.3,59.9)$ & $15.8(14.9,16.7)$ & $41.8(40.5,43.1)$ & $48.7(47.4,50.0)$ & $3.6(3.1,4.1)$ \\
\hline Overall & $0.9(0.8,1.0)$ & $1.2(1.1,1.4)$ & $23.3(22.7,23.9)$ & $6.5(6.2,6.8)$ & $26.8(26.2,27.4)$ & $31.8(31.2,32.4)$ & $1.5(1.3,1.7)$ \\
\hline
\end{tabular}

$\mathrm{CVD}=$ cardiovascular disease, $\mathrm{CHD}=$ coronary heart disease, $\mathrm{CI}=$ confidence interval.

Table 2

The weighted levels of CVD risk factors stratified by self-reported CHD and stroke among Nanjing sample $(n=40,896)$. 


\begin{tabular}{|c|c|c|c|c|c|c|c|}
\hline \multirow{2}{*}{$\begin{array}{l}\text { Risk factors } \\
\text { mean } \pm \mathrm{SD}\end{array}$} & \multirow[t]{2}{*}{ Total } & \multicolumn{3}{|c|}{ CHD } & \multicolumn{3}{|c|}{ Stroke } \\
\hline & & Yes & No & $P$ value & Yes & No & $P$ value \\
\hline Body mass index (BMI), $\mathrm{kg} / \mathrm{m}^{2}$ & $23.0 \pm 3.2$ & $23.6 \pm 3.5$ & $23.0 \pm 3.2$ & $<0.001$ & $24.0 \pm 3.4$ & $23.0 \pm 3.2$ & $<0.001$ \\
\hline Systolic blood pressure (SBP), mmHg & $121.3 \pm 15.5$ & $131.6 \pm 21.0$ & $121.1 \pm 15.4$ & $<0.001$ & $131.1 \pm 16.8$ & $121.1 \pm 15.4$ & $<0.001$ \\
\hline Diastolic blood pressure (DBP), $\mathrm{mmHg}$ & $77.9 \pm 10.0$ & $85.1 \pm 13.6$ & $77.9 \pm 9.8$ & $<0.001$ & $84.0 \pm 9.4$ & $77.8 \pm 9.8$ & $<0.001$ \\
\hline Fasting plasma glucose (FPG), mmol/L & $5.2 \pm 1.5$ & $7.8 \pm 1.7$ & $5.2 \pm 1.4$ & $<0.001$ & $6.7 \pm 1.4$ & $5.2 \pm 1.4$ & $<0.001$ \\
\hline Total cholesterol (TC), mmol/L & $4.7 \pm 1.4$ & $9.3 \pm 1.6$ & $4.6 \pm 1.3$ & $<0.001$ & $7.4 \pm 1.0$ & $4.6 \pm 1.1$ & $<0.001$ \\
\hline Triglycerides (TG), mmol/L & $1.6 \pm 0.9$ & $4.9 \pm 1.9$ & $1.6 \pm 0.7$ & $<0.001$ & $2.6 \pm 0.4$ & $1.6 \pm 0.9$ & $<0.001$ \\
\hline $\begin{array}{l}\text { Low-density lipoprotein cholesterol (LDL-C), } \\
\mathrm{mmol} / \mathrm{L}\end{array}$ & $2.6 \pm 0.9$ & $2.7 \pm 1.0$ & $2.6 \pm 0.9$ & $<0.001$ & $2.7 \pm 1.0$ & $2.6 \pm 0.9$ & $<0.001$ \\
\hline $\begin{array}{l}\text { High-density lipoprotein cholesterol (HDL-C), } \\
\mathrm{mmol} / \mathrm{L}\end{array}$ & $1.5 \pm 0.5$ & $1.4 \pm 0.5$ & $1.5 \pm 0.5$ & $<0.001$ & $1.4 \pm 0.7$ & $1.5 \pm 0.5$ & $<0.001$ \\
\hline
\end{tabular}

$\mathrm{CVD}=$ cardiovascular disease, $\mathrm{CHD}=$ coronary heart disease, $\mathrm{SD}=$ standard deviation.

Table 3

The relationship between CVD risk factor levels and self-reported CHD and stroke among Nanjing sample $(n=40,896)$.

\begin{tabular}{|c|c|c|c|c|}
\hline \multirow{2}{*}{\multicolumn{2}{|c|}{ Risk factors }} & \multicolumn{3}{|c|}{ Adjusted $O R$ and $95 \% C I$} \\
\hline & & Model $1^{a}$ & Model $2^{\text {b }}$ & Model $3^{c}$ \\
\hline \multicolumn{5}{|l|}{$\overline{\mathrm{HD}}$} \\
\hline Body mass index (BMI), $\mathrm{kg} / \mathrm{m}^{2}$ & & $1.147(1.115,1.179)^{*}$ & $1.125(1.092,1.160)^{*}$ & $\begin{array}{c}1.086 \underset{*}{(1.051,1.122)} \\
*\end{array}$ \\
\hline Systolic blood pressure (SBP), mmHg & & $1.027(1.022,1.032) *$ & $\begin{array}{c}1.005(1.004,1.007) \\
*\end{array}$ & $\begin{array}{c}1.004(1.001,1.007) \\
\dagger\end{array}$ \\
\hline Diastolic blood pressure (DBP), $\mathrm{mmHg}$ & & $1.028(1.021,1.035) *$ & $\begin{array}{c}1.004(1.001,1.007) \\
\dagger\end{array}$ & $1.003(0.999,1.008)$ \\
\hline Fasting plasma glucose (FPG), mmol/L & & $1.238(1.200,1.278) *$ & $\begin{array}{c}1.088(1.057,1.120) \\
*\end{array}$ & $\begin{array}{c}1.053(1.005,1.105) \\
\dagger\end{array}$ \\
\hline Total cholesterol (TC), mmol/L & & $1.109(1.009,1.219) \dagger$ & $1.041(0.920,1.179)$ & $0.963(0.842,1.102)$ \\
\hline Triglycerides (TG), mmol/L & & $\begin{array}{c}1.149(1.093,1.208) * \mathrm{p}<0.01 \mathrm{p}<0.01 \\
\mathrm{p}<0.01\end{array}$ & $1.045(0.979,1.116)$ & $\begin{array}{c}1.074(1.002,1.152) \\
\dagger\end{array}$ \\
\hline $\begin{array}{l}\text { Low-density lipoprotein cholesterol } \\
\mathrm{nmol} / \mathrm{L}\end{array}$ & (LDL-C), & $1.070(0.941,1.216)$ & $0.916(0.781,1.073)$ & $0.854(0.724,1.009)$ \\
\hline $\begin{array}{l}\text { High-density lipoprotein cholesterol } \\
\text { nmol/L } \\
\text { itroke }\end{array}$ & (HDL-C), & $0.604(0.460,0.793) * \mathrm{p}<0.01$ & $\begin{array}{c}0.711(0.543,0.931) \\
\dagger\end{array}$ & $\begin{array}{c}0.690(0.524,0.909) \\
\dagger\end{array}$ \\
\hline Body mass index (BMI), kg/m² & & $1.127(1.108,1.147){ }^{*}$ & $\begin{array}{l}1.108(1.087 \\
1.129)^{*}\end{array}$ & $1.062(1.040,1.084)^{*}$ \\
\hline Systolic blood pressure (SBP), mmHg & & $1.029(1.025,1.033){ }^{*}$ & $\begin{array}{l}1.007(1.005 \\
1.008)^{*}\end{array}$ & $1.005(1.003,1.007){ }^{*}$ \\
\hline Diastolic blood pressure (DBP), $\mathrm{mmHg}$ & & $1.027(1.021,1.034)^{*}$ & $\begin{array}{l}1.004(1.002 \\
1.006)^{*}\end{array}$ & $1.003(1.000,1.006)$ \\
\hline Fasting plasma glucose (FPG), mmol/L & & $1.168(1.133,1.204){ }^{*}$ & $\begin{array}{l}1.111(1.089 \\
1.134)^{*}\end{array}$ & $1.064(1.032,1.096){ }^{*}$ \\
\hline Total cholesterol (TC), mmol/L & & $1.135(1.074,1.200)^{*}$ & $1.027(0.949,1.111)$ & $0.927(0.853,1.008)$ \\
\hline Triglycerides (TG), mmol/L & & $1.088(1.043,1.135)^{*}$ & $0.977(0.925,1.032)$ & $1.003(0.945,1.066)$ \\
\hline $\begin{array}{l}\text { Low-density lipoprotein cholesterol } \\
\mathrm{nmol} / \mathrm{L}\end{array}$ & $(\mathrm{LDL}-\mathrm{C})$ & $1.176(1.090,1.268)^{*}$ & $1.036(0.939,1.143)$ & $0.961(0.867,1.066)$ \\
\hline $\begin{array}{l}\text { High-density lipoprotein cholesterol } \\
\mathrm{nmol} / \mathrm{L}\end{array}$ & (HDL-C), & $0.913(0.796,1.047)$ & $1.003(0.878,1.146)$ & $0.977(0.853,1.120)$ \\
\hline
\end{tabular}


$\mathrm{J}=$ cardiovascular disease, $\mathrm{CHD}=$ coronary heart disease, $\mathrm{OR}=$ odds ratio, $\mathrm{CI}=$ confidence interval.

odel 1 was adjusted for age only.

odel 2 was adjusted for age plus all other four CVD risk factor values.

odel 3 was adjusted for all variables in model 2 plus marriage, residence, SES, family history of CVDs, physical activity and dietary habits.

) $1,+p<0.05$.

Table 4

The relationship between CVD risk factor and self-reported CHD and stroke among Nanjing sample $(n=40,896)$.

\begin{tabular}{|c|c|c|c|}
\hline \multirow[t]{2}{*}{ Risk factors } & \multicolumn{3}{|c|}{ Adjusted $O R$ and $95 \% C I$} \\
\hline & Model $1^{a}$ & Model $2^{b}$ & Model $3^{c}$ \\
\hline \multicolumn{4}{|l|}{ CHD } \\
\hline \multicolumn{4}{|l|}{ Men } \\
\hline Hypertension & $3.888(2.638 \sim 5.729)^{*}$ & $3.840(2.825 \sim 5.219)^{*}$ & $3.192(2.321 \sim 4.391)^{*}$ \\
\hline Diabetes & $4.324(3.269 \sim 5.719){ }^{*}$ & $3.241(2.436 \sim 4.312)^{*}$ & $2.547(1.793 \sim 3.617)^{*}$ \\
\hline Dyslipidemia & $3.142(2.400 \sim 4.112)^{*}$ & $2.623(1.988 \sim 3.462)^{*}$ & $1.936(1.395 \sim 2.686)^{*}$ \\
\hline Overweight or obesity & $1.029(0.750 \sim 1.260)$ & $0.928(0.779 \sim 1.222)$ & $0.851(0.674 \sim 1.395)$ \\
\hline Current smoking & $1.524(1.273 \sim 1.825)^{*}$ & $1.218(1.016 \sim 1.460) \dagger$ & $1.050(0.811 \sim 1.360)$ \\
\hline \multicolumn{4}{|l|}{ Women } \\
\hline Hypertension & $3.247(2.410 \sim 4.375) *$ & $2.769(2.042 \sim 3.753)^{*}$ & $3.231(2.254 \sim 4.633) *$ \\
\hline Diabetes & $2.448(1.825 \sim 3.284)^{*}$ & $1.940(1.440 \sim 2.614)^{*}$ & $1.799(1.255 \sim 2.578){ }^{*}$ \\
\hline Dyslipidemia & $2.011(1.553 \sim 2.602){ }^{*}$ & $1.692(1.302 \sim 2.197)^{*}$ & $1.595(1.170 \sim 2.174)^{*}$ \\
\hline Overweight or obesity & $1.466(1.134 \sim 1.897)^{*}$ & $1.137(0.874 \sim 1.479)$ & $1.103(0.809 \sim 1.504)$ \\
\hline Current smoking & 1.199 (0.490 2.932) & $1.151(0.472 \sim 2.806)$ & $1.083(0.444 \sim 2.644)$ \\
\hline \multicolumn{4}{|l|}{ Stroke } \\
\hline \multicolumn{4}{|l|}{ Men } \\
\hline Hypertension & $5.587(4.239 \sim 7.363)^{*}$ & $5.505(3.982 \sim 7.610)^{*}$ & $4.779(3.599 \sim 6.348){ }^{*}$ \\
\hline Diabetes & $3.262(2.586 \sim 4.114)^{*}$ & $2.374(1.872 \sim 3.009)^{*}$ & $2.385(1.812 \sim 3.138)^{*}$ \\
\hline Dyslipidemia & $2.529(2.047 \sim 3.124)^{*}$ & $2.004(1.611 \sim 2.493)^{*}$ & $1.659(1.290 \sim 2.133){ }^{*}$ \\
\hline Overweight or obesity & $1.288(1.047 \sim 1.586) \dagger$ & $0.838(0.675 \sim 1.040)$ & $0.830(0.648 \sim 1.062)$ \\
\hline Current smoking & 1.168 (0.695 1.055) & $1.151(0.971 \sim 1.365)$ & $1.028(0.866 \sim 1.222)$ \\
\hline \multicolumn{4}{|l|}{ Women } \\
\hline Hypertension & $4.767(3.460 \sim 6.568)^{*}$ & $4.388(3.320 \sim 5.798)^{*}$ & $3.852(2.902 \sim 5.114){ }^{*}$ \\
\hline Diabetes & $1.932(1.476 \sim 2.529)^{*}$ & $1.472(1.120 \sim 1.935)^{*}$ & $1.376(1.060 \sim 1.900) \dagger$ \\
\hline Dyslipidemia & $2.263(1.804 \sim 2.840)^{*}$ & $1.916(1.522 \sim 2.412)^{*}$ & $1.462(1.125 \sim 1.898)^{*}$ \\
\hline Overweight or obesity & $1.426(1.139 \sim 1.784){ }^{*}$ & $1.075(0.855 \sim 1.351)$ & $0.986(0.759 \sim 1.281)$ \\
\hline Current smoking & $1.056(0.555 \sim 2.009)$ & $1.151(0.601 \sim 2.204)$ & $0.972(0.435 \sim 2.172)$ \\
\hline
\end{tabular}

$\mathrm{CVD}=$ cardiovascular disease, $\mathrm{CHD}=$ coronary heart disease, $\mathrm{OR}=$ odds ratio, $\mathrm{CI}=$ confidence interval.

a Model 1 was adjusted for age only.

${ }^{\mathrm{b}}$ Model 2 was adjusted for age plus all other four CVD risk factors.

${ }^{\mathrm{C}}$ Model 3 was adjusted for all variables in model 2 plus marriage, residence, SES, family history of CVDs,

physical activity and dietary habits.

${ }^{*} p<0.01,+p<0.05$

Table 5 
The relationship between numbers of CVD risk factors and self-reported CHD and stroke among Nanjing sample (n=40,896).

\begin{tabular}{|c|c|c|}
\hline \multirow[t]{2}{*}{ Category } & \multicolumn{2}{|c|}{ Adjusted $O R$ and $95 \% C I$} \\
\hline & Model $1^{a}$ & Model $2^{b}$ \\
\hline \multicolumn{3}{|l|}{$\mathrm{CHD}$} \\
\hline \multicolumn{3}{|l|}{ Men } \\
\hline 0 risk factor & Ref & Ref \\
\hline 1 risk factor & $2.034(0.979 \sim 4.228)$ & $1.324(0.609 \sim 2.876)$ \\
\hline 2 risk factors & $3.394(1.684 \sim 6.840)^{*}$ & $2.654(1.283 \sim 5.487){ }^{*}$ \\
\hline$\geq 3$ risk factors & $6.197(3.128 \sim 12.274)^{*}$ & $4.449(2.195 \sim 9.021)^{*}$ \\
\hline \multicolumn{3}{|l|}{ Women } \\
\hline 0 risk factor & Ref & Ref \\
\hline 1 risk factor & $3.673(1.836 \sim 7.350){ }^{*}$ & $3.007(1.698 \sim 5.327)^{*}$ \\
\hline 2 risk factors & $4.480(2.228 \sim 9.011)^{*}$ & $3.564(2.009 \sim 6.322){ }^{*}$ \\
\hline$\geq 3$ risk factors & $9.730(4.885 \sim 15.382)^{*}$ & $8.413(4.808 \sim 14.721)^{*}$ \\
\hline \multicolumn{3}{|l|}{ Stroke } \\
\hline \multicolumn{3}{|l|}{ Men } \\
\hline 0 risk factor & Ref & Ref \\
\hline 1 risk factor & $3.467(1.470 \sim 8.176)^{*}$ & $3.171(1.569 \sim 6.405)^{*}$ \\
\hline 2 risk factors & $4.867(2.098 \sim 11.294)^{*}$ & $4.059(2.037 \sim 8.088)^{*}$ \\
\hline$\geq 3$ risk factors & $11.352(4.984 \sim 18.857) *$ & $9.619(4.915 \sim 15.825)^{*}$ \\
\hline \multicolumn{3}{|l|}{ Women } \\
\hline 0 risk factor & Ref & Ref \\
\hline 1 risk factor & $1.595(0.970 \sim 2.623)$ & $1.645(0.953 \sim 2.839)$ \\
\hline 2 risk factors & $4.080(2.582 \sim 6.449){ }^{*}$ & $3.991(2.399 \sim 6.638){ }^{*}$ \\
\hline$\geq 3$ risk factors & $6.462(4.078 \sim 10.238)^{*}$ & $5.685(3.394 \sim 9.524){ }^{*}$ \\
\hline
\end{tabular}

vascular disease, $\mathrm{CHD}=$ coronary heart disease, $\mathrm{OR}=$ odds ratio, $\mathrm{CI}=$ confidence interval.

as adjusted for age only.

as adjusted for age plus marriage, residence, SES, family history of CVDs, physical activity habits.

\section{Figures}




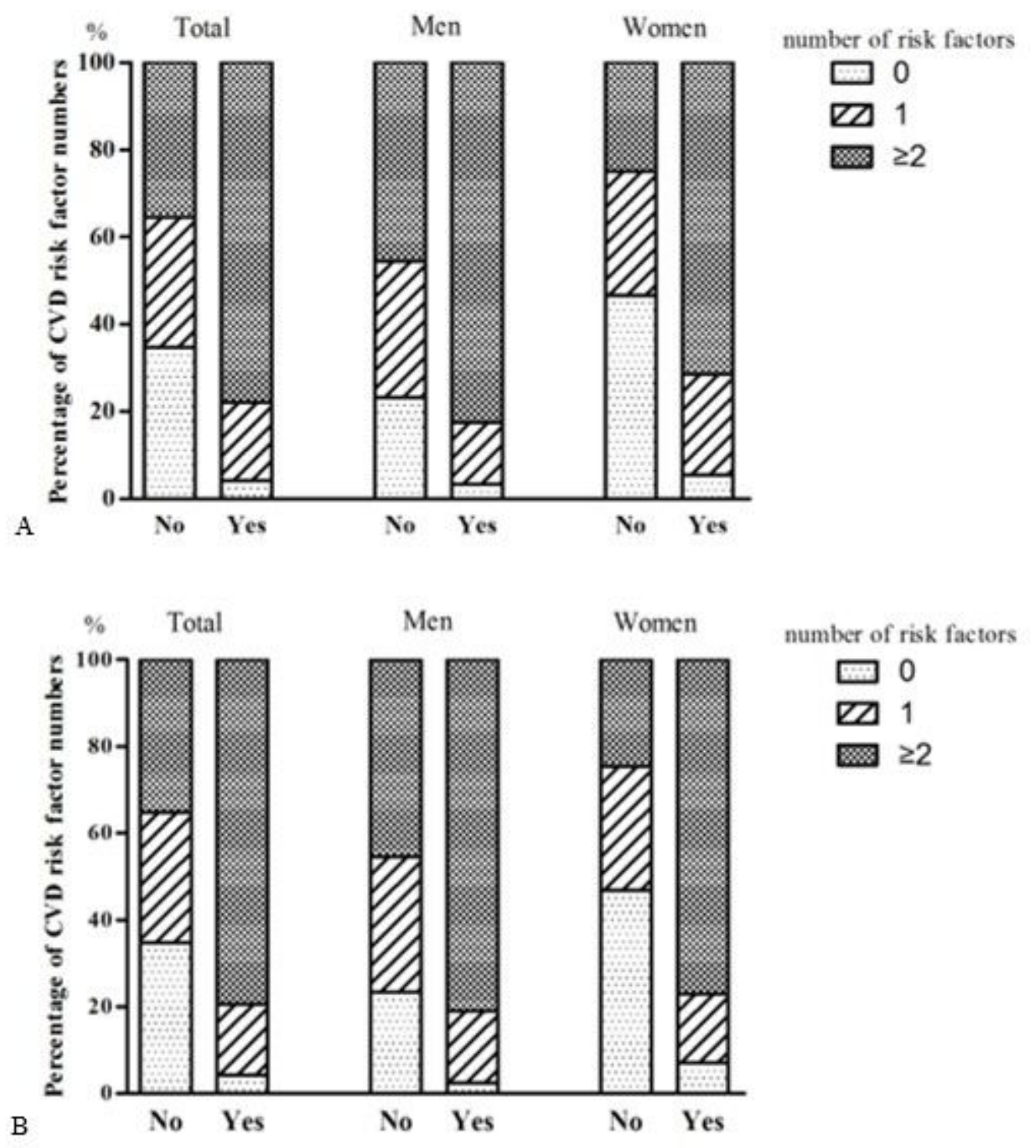

Figure 1

(A) Percentage of cardiovascular disease (CVD) risk factor numbers by self-reported coronary heart disease (CHD) among Nanjing sample and different genders. No: participants without CHD, Yes: participants with CHD. (B) Percentage of CVD risk factor numbers by self-reported stroke among Nanjing sample and different genders. No: participants without stroke; Yes: participants with stroke. 